\title{
Seepage Safety Assessment for Baiyun Hydropower Station
}

\author{
Wei Liang ${ }^{1, a}$, Bo Li ${ }^{2, b}$ \\ ${ }^{1}$ Engineering Safety and Disaster Prevention Department, Changjiang River Scientific Research \\ Institute, China \\ ${ }^{2}$ Engineering Safety and Disaster Prevention Department, Changjiang River Scientific Research \\ Institute, China \\ a114676818@qq.com, b14175855@qq.com
}

\begin{abstract}
Keywords: Baiyun Hydropower Station the seepage property Safety Assessment
\end{abstract} Abstract. Baiyun Hydropower Station is located in Chengbu County, Hunan Province, which is 5km from the county town. By combining the analysis of monitoring data of leakage amount of above-mentioned dam and the numerical calculation results, it is concluded that, there are serious leakage at the lower face of left bank and near the toe board, which results in the potential safety hazard of major seepage flow. In accordance with "Guidelines on Dam Safety Evaluation", the seepage property of Baiyun hydropower dam is rated as Grade " $\mathrm{C}$ ".

\section{Project Overview}

Baiyun Hydropower Station is located in Chengbu County, Hunan Province, which is $5 \mathrm{~km}$ from the county town. It is located inside the canyon at the upstream of $\mathrm{Wu}$ River, and is the first grade tributary flowing of Yuan River. The main benefit objective of Baiyun Hydropower Station is electricity generation, with the average annual energy generation of 0.1168 billion $\mathrm{kWh}$. The dam is of rolled rockfill dam with reinforced concrete face, and the dam is $120 \mathrm{~m}$ in height. The controlled drainage areas is $556 \mathrm{~km}^{2}$, and the mean annual runoff is of 0.533 billion $\mathrm{m}^{3}$. The normal water level of

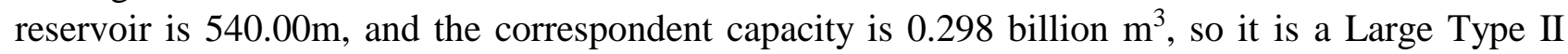
reservoir.

\section{Analysis of Monitoring Data of Seepage Flow}

Analysis of monitoring data of seepage amount of dam

A concrete cut-off wall and a shaft of measuring weir at the downstream dam toe, for the observation of seepage amount of dam. Within 10 years after the reservoir impoundment power generation of dam, the observed value of leakage fell within the range of normal value; after May 2008, the leakage amount started to increased, and it reached 105L/s. In 2008, it reached 200L/s, and in September 2008, it reached 300L/s, and in February 2009, it reached 400L/s, and in August 2009, it reached $500 \mathrm{~L} / \mathrm{s}$, and in October, it reached $521 \mathrm{~L} / \mathrm{s}$, and in October, 2010, it was close to $800 \mathrm{~L} / \mathrm{s}$. In May 2012, the leakage amount was increased to $1000 \mathrm{~L} / \mathrm{s}$, and it reached the maximum value of $1248.71 \mathrm{~L} / \mathrm{s}$ in September. The changing curves of reservoir water level and leakage amount are shown as Figure 2.1.1. 


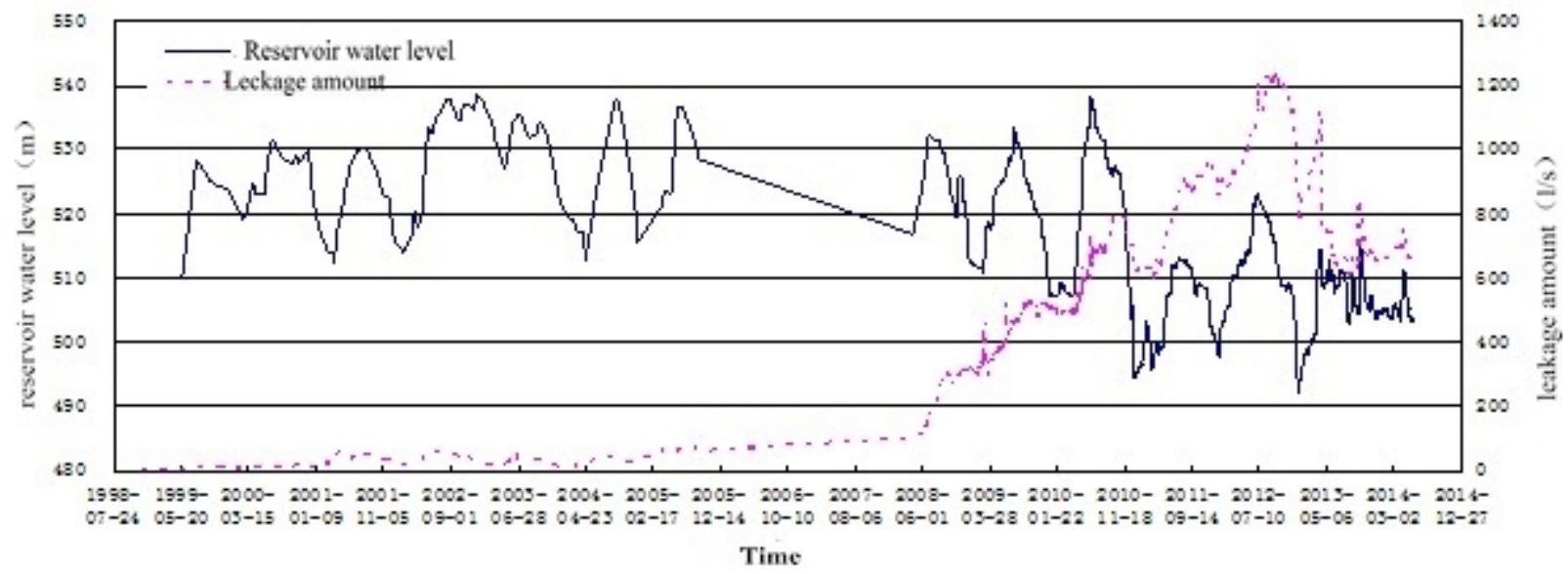

Figure 2.1.1 Varying curve of dam leakage amount of Baiyun Hydropower Station

From the overall varying pattern of leakage amount, the leakage trend of dam keeps on increasing. From 2008 to 2012, the leakage amount at the same reservoir water level display a trend of continuous increase. After the end of year 2010, every time the reservoir water level increases, the leakage amount will greatly be increased, while only when there are great reductions in the reservoir water level (lower than the level of dead water), will the leakage amount be significantly reduced.

\section{Analysis of Monitoring Data of Around-the-dam Seepage Flow}

The measuring points of around-the-dam seepage flow of Baiyun Hydropower Station are arranged as follows: 1\# hole and 3 \# hole on the left bank of dam, and 3\# hole and $4 \#$ hole on the right bank of dam. From Figure2.2.1 and Figure 2.2.2, it can be seen that the measured value of around-the-dam seepage flow of $1 \#$ on the left bank is higher than the reservoir water level, which is mainly influenced by the nearby underground water. The changing curve of measured values of around-the-dam seepage flows at $1 \#$ hole and 2\# hole on the right bank, and 3\# hole and 4\# hole on the right bank approximates a straight line, which has very little relations with reservoir water level and rainfall, and almost free of influence of reservoir water level and rainfall. It indicates that the reliability of measured values of four measuring points of around-the-dam seepage needs to be further verified.

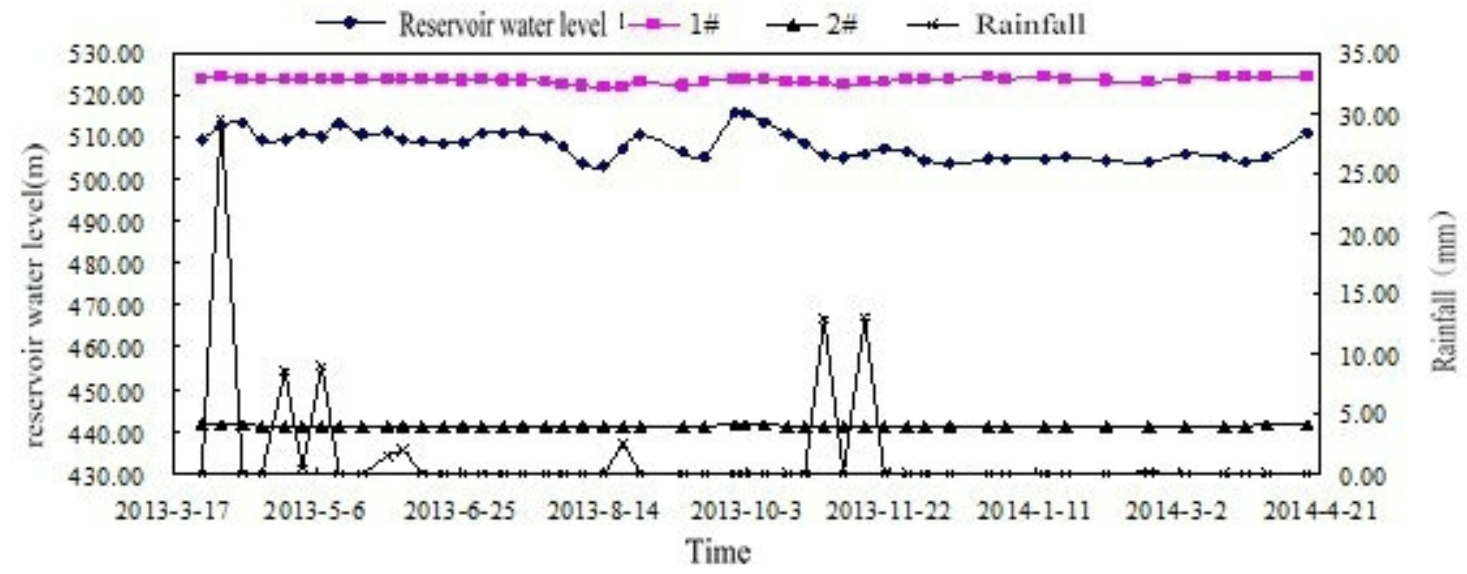

Figure 2.2.1 hydrograph of measured values of around-the-dam seepage flows at 1\# hole and 2\# hole on the left bank. 


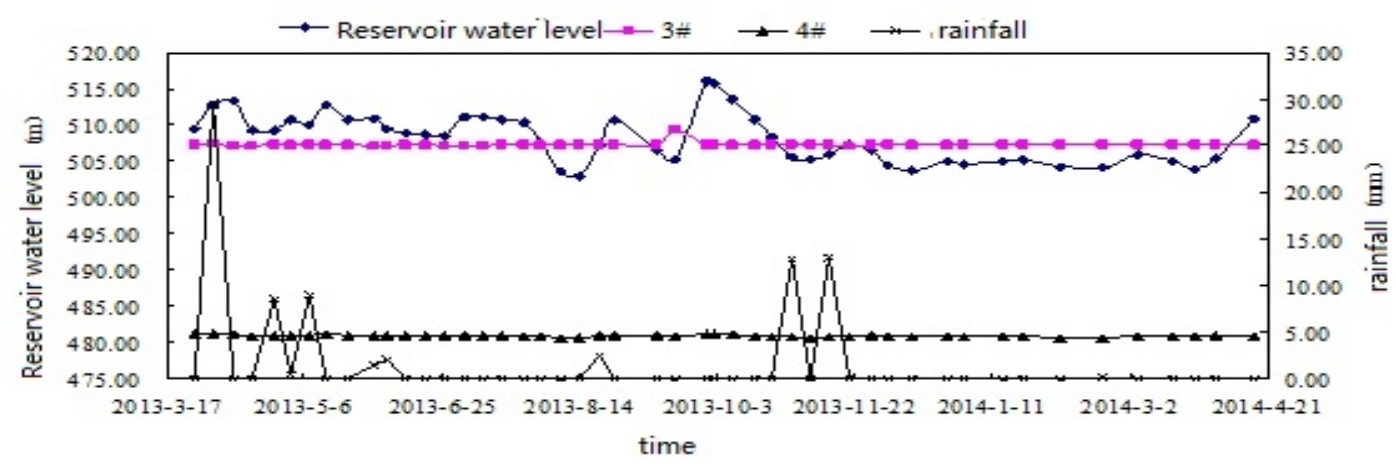

Figure 2.2.2 Hydrograph of measured values of around-the-dam seepage flows at $3 \#$ hole and $4 \#$ hole on the right bank.

\section{Calculation and Analysis of Seepage Flow Values}

The calculation and analysis of steady seepage are performed for the concrete faced rockfill dam of Baiyun, and there are two purposes of doing this: the first one is to verify the dam seepage line and seepage flow under normal condition, and the second one is to systematically evaluate the distribution characteristics of seepage field and the seepage control effect of anti-seepage system of dam. The seepage numerical calculation is made on the assumption that the concrete face and the waterstop remain undamaged.

\section{Fundamental principles of calculation of seepage flow}

Darcy law is one of most fundamental equations adopted in researches of seepage flow, and for three-dimensional flow of isotropic medium, Darcy law is as follows:

$$
v=k J=-k g r a d H
$$

Under 1D condition, ${ }^{v}$ is seepage velocity, $J=-\operatorname{grad} H$ is hydraulic gradient, and both of them are scalar quantities; under 3D conditions, $v$ is velocity vector, and $J=-\operatorname{gradH}$ is gradient vector. For isotropic medium, $k$ is permeability coefficient (scalar quantity), for anisotropic medium, $k$ is permeability tensor. For the seepage flow of anisotropic medium, the above formula can be written as:

$$
\left\{\begin{array}{l}
v_{x}=-k_{x x} \frac{\partial H}{\partial x}-k_{x y} \frac{\partial H}{\partial y}-k_{x z} \frac{\partial H}{\partial z} \\
v_{y}=-k_{y x} \frac{\partial H}{\partial x}-k_{y y} \frac{\partial H}{\partial y}-k_{y z} \frac{\partial H}{\partial z} \\
v_{z}=-k_{z x} \frac{\partial H}{\partial x}-k_{z y} \frac{\partial H}{\partial y}-k_{z z} \frac{\partial H}{\partial z}
\end{array}\right.
$$

Assume that the fluid is homogeneously incompressible medium, for fluid micro-body, the water volumes that flows into and outflows during the period of time is equal. Therefore, the continuity equation of steady seepage can be deduced:

$$
\frac{\partial v_{x}}{\partial x}+\frac{\partial v_{y}}{\partial y}+\frac{\partial v_{z}}{\partial z}-Q=0
$$

Where, $Q$ is micro-body endogenesis. The equation of continuity is the specific application of law of conservation of mass in the issue of seepage flow, and the formula suggested that, in the flowing process, the mass of fluid neither increases nor decreases.

Make formula (2) and (3) basic simultaneous, and the basic differential equations that describes anisotropic steady seepage field is obtained: 


$$
\begin{aligned}
& \frac{\partial}{\partial x}\left(-k_{x x} \frac{\partial H}{\partial x}-k_{x y} \frac{\partial H}{\partial y}-k_{x z} \frac{\partial H}{\partial z}\right)+\frac{\partial}{\partial y}\left(-k_{y x} \frac{\partial H}{\partial x}-k_{y y} \frac{\partial H}{\partial y}-k_{y z} \frac{\partial H}{\partial z}\right)+ \\
& \frac{\partial}{\partial z}\left(-k_{z x} \frac{\partial H}{\partial x}-k_{z y} \frac{\partial H}{\partial y}-k_{z z} \frac{\partial H}{\partial z}\right)-Q=0
\end{aligned}
$$

If $\mathrm{x}, \mathrm{y}$ and $\mathrm{z}$ are main direction, and the endogenesis is 0 , then the above equation can be written as:

$$
\frac{\partial}{\partial x}\left(k_{x} \frac{\partial H}{\partial x}\right)+\frac{\partial}{\partial y}\left(k_{y} \frac{\partial H}{\partial y}\right)+\frac{\partial}{\partial z}\left(k_{z} \frac{\partial H}{\partial z}\right)=0
$$

where, $k_{x}, k_{y}$ and $k_{z}$ are permeability coefficients at three main directions of seepage flows. when $k_{x}=k_{y}=k_{z}$, for isotropic seepage flows, Laplace equation can be deduced:

$$
\frac{\partial^{2} H}{\partial x^{2}}+\frac{\partial^{2} H}{\partial y^{2}}+\frac{\partial^{2} H}{\partial z^{2}}=0
$$

For steady seepage, the boundary conditions are the definite condition of basic differential equations.

Generally speaking, there are three types of boundary conditions of seepage flow. The boundary of the first type (Dirichlet boundary condition): the hydraulic head of boundary $\left(\Gamma_{1}\right)$ is known, that is,

$$
\Gamma_{1}=\phi(x, y, z)
$$

The boundary of the second type (Neumann boundary conditions): the flow of boundary $\left(\Gamma_{2}\right)$ is known, that is,

$$
\left.k \frac{\partial H}{\partial n}\right|_{\Gamma_{2}}=q(x, y, z)
$$

The boundary of the third type: the boundaries of free surface $\left(\Gamma_{3}\right)$ and overflow surface $\left(\Gamma_{4}\right)$, that is.

$\begin{array}{ll}\text { free surface } \Gamma_{3}: & \left\{\begin{array}{l}\left.H\right|_{\Gamma_{3}}=Z(x, y) \\ \left.k \frac{\partial H}{\partial n}\right|_{\Gamma_{3}}=0\end{array}\right. \\ \text { overflow surface } \Gamma_{4}: \quad\left\{\begin{array}{l}\left.H\right|_{\Gamma_{4}}=Z(x, y) \\ \left.k \frac{\partial H}{\partial n}\right|_{\Gamma_{4}} \leq 0\end{array}\right.\end{array}$

By combining the basic differential equations and the seepage flow boundary conditions, the steady seepage field can be calculated.

\section{Calculation Model and Computational Condition}

\section{(1) Calculation model}

The finite element method is mainly adopted to construct a finite element model that meets the simulation precision and the requirement of engineering practice, and a reasonable steady seepage analysis is performed for the dam body, with the finite element model and material partition as shown in Figure 3.2.1. The finite element model is a pseudo three-dimensional based on typical cross-section dimension, which composed of 5712 nodes and 2769 units. By performing calculation of steady seepage for the typical section of dam body, the regularities of distribution of seepage field is analyzed. 


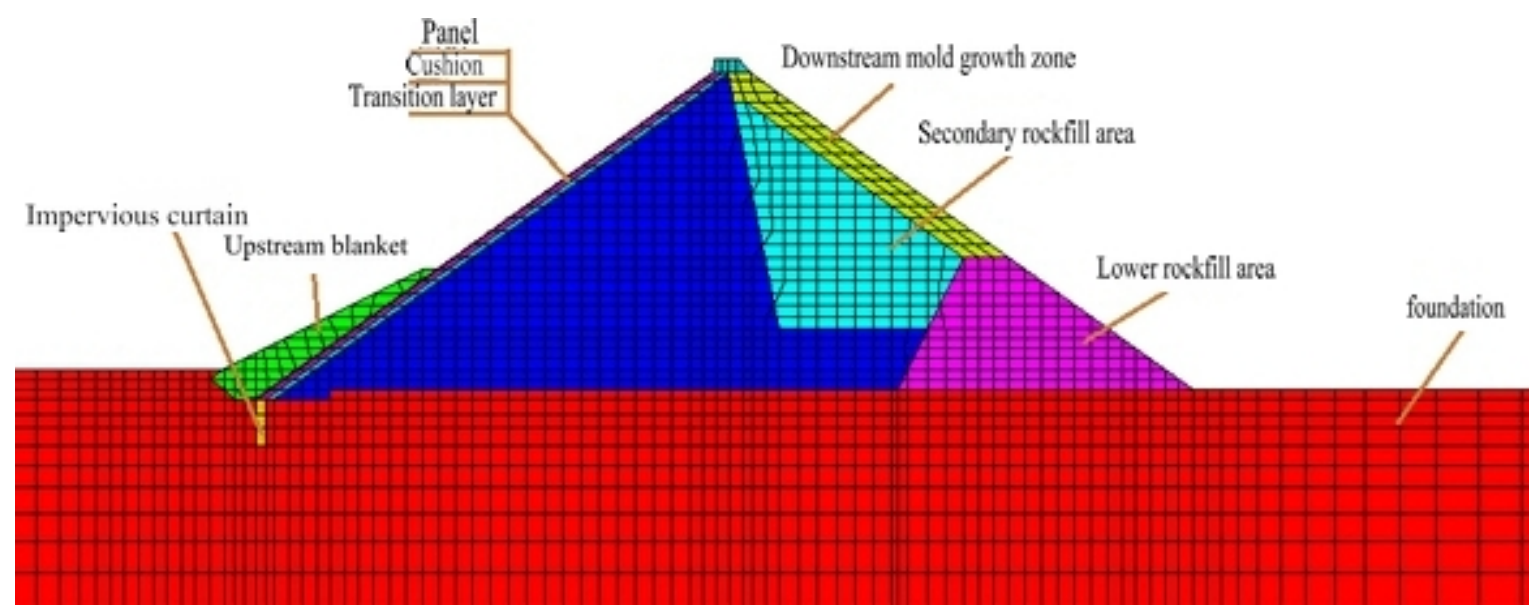

Figure 3.2.1 Typical section's finite element model and material partition

(2) Calculation parameters

According to different lithology of parent rocks that the dam is made of in different districts, the permeability coefficients of different materials are determined by referring to the lithology ad parameters of relevant projects, and the values of permeability coefficients of different materials are as per Table 3.2.1.

Table 3.2.1 values of permeability coefficient of materials.

\begin{tabular}{lc}
\hline Name & $\begin{array}{c}\text { permeability } \\
\text { coefficient }(\mathrm{m} / \mathrm{s})\end{array}$ \\
\hline foundation & $3 \times 10^{-6}$ \\
upstream blanket & $2.7 \times 10^{-3}$ \\
anti-seepage curtain & $1 \times 10^{-9}$ \\
face & $2.4 \times 10^{-9}$ \\
cushion & $1 \times 10^{-4}$ \\
transition layer & $3.4 \times 10^{-3}$ \\
main rockfill area & $4.3 \times 10^{-4}$ \\
secondary rockfill area & $3.4 \times 10^{-4}$ \\
downstream rockfill area & $2.2 \times 10^{-3}$ \\
downstream plasticatio & $4.3 \times 10^{-4}$ \\
area & \\
\hline
\end{tabular}

(3) Calculation of working condition

For the normal operation condition, the normal pool level is taken as $540.00 \mathrm{~m}$ at the upstream, while the normal tail level is taken as $434 \mathrm{~m}$ at the downstream.

\section{Calculation Results and Analysis}

Under normal operation condition of reservoir, the equivalent hydraulic head line of typical section of dam, the contour line of pore water pressure and the seepage amount are shown as Figure 3.3.1 3.3.2. From Figure 3.3.1, it can be found that, the distribution of equipotential line of seepage pressure at the parts of face and anti-seepage curtain is relatively concentrated, which radiates all around from the dam heel area, and the hydraulic head decreases sharply. The subsection of downstream equipotential lines is scattered, and the hydraulic head decreases mildly. This indicated that, under the normal operation condition, the face and the anti-seepage curtain has the seepage-proofing function. 


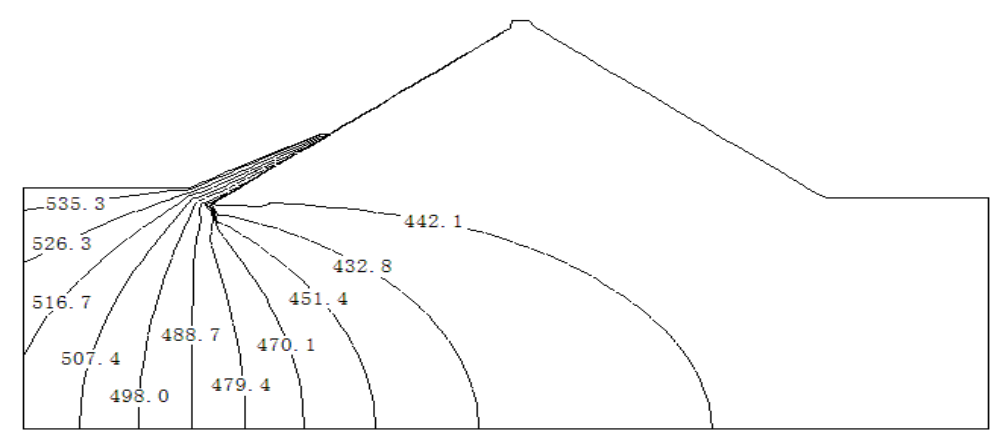

Figure 3.3.1 Equivalent hydraulic head lines of typical sections(unit: $\mathrm{m}$ )

From Figure 3.3.2, the pore water pressure at the face and the anti-seepage curtain parts significantly decreases, and the free surface of free surface, that is, saturation line, decreases sharply in the concrete face, while it decreases mildly in the main and secondary rockfill areas and the downstream rockfill area. It oozes at the elevation of river water level of the downstream rock mass of dam body. The distribution characteristics of free surface inside the material partition of dam body meets two basic geometric properties, that is, smooth continuity and back-bending impossibility.

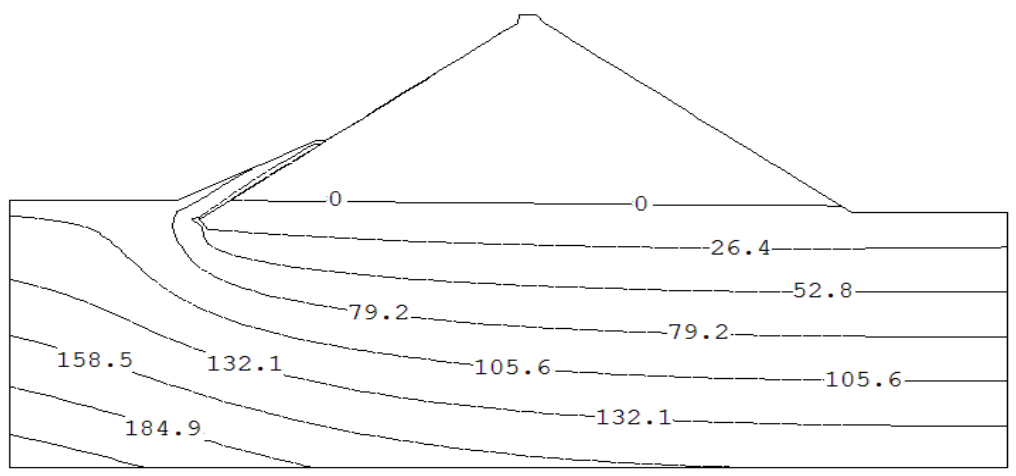

Figure 3.3.2 contour line of pore water pressure of typical section (unit: $\mathrm{m}$ )

From Figure 3.3.3, we can find that, under normal operation, the face and the toe board have relatively small leakage amount. The flow at the bottom of upstream anti-seepage curtain and near the water surface is the greatest, and the seepage flow velocity of dam body gradually decreases from the dam crest to dam base, and the flow line has relatively large degree of crook at the upstream dam heel area, and the seepage amount at the dam slope close to the water surface is the greatest, which basically meets the actual seepage flow situations. The unit rate of seepage flow of dam section is $14.2 \mathrm{~m}^{3} / \mathrm{d}$. 


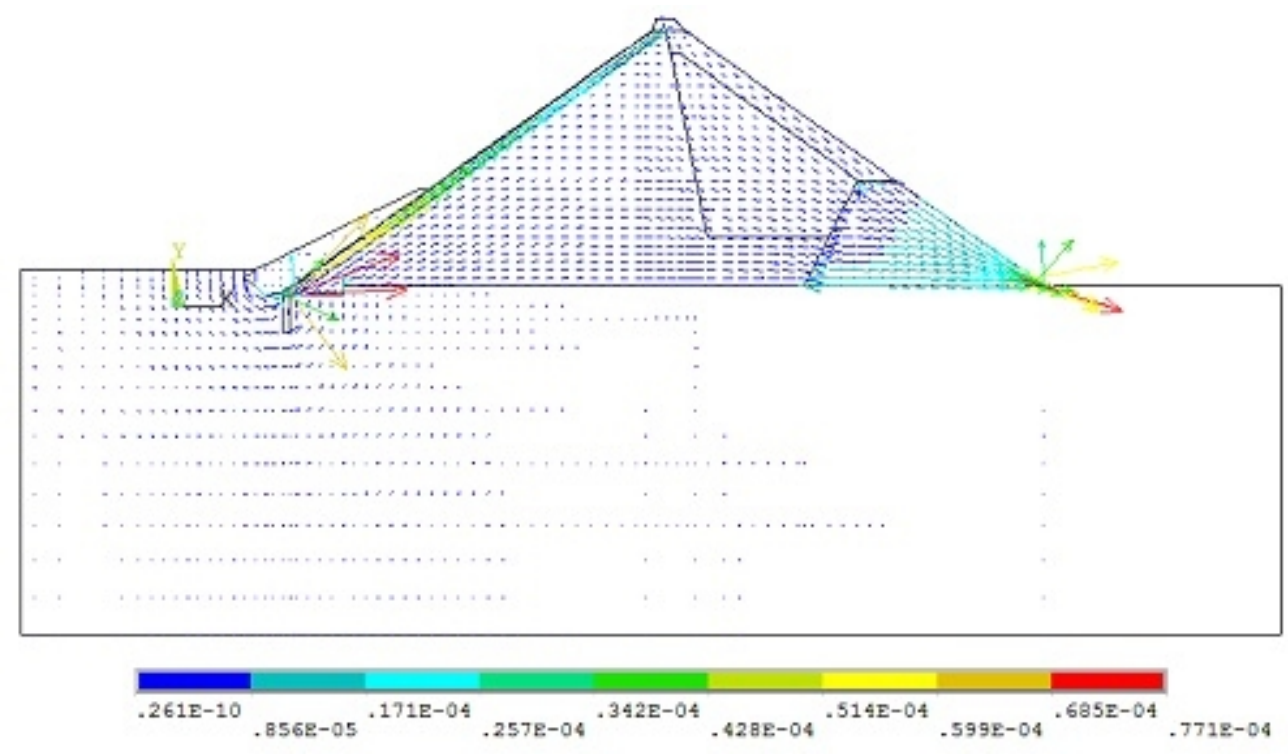

Figure 3.3.3 Velocity vector of typical section

\section{Conclusion and Suggestion}

By analyzing the current monitoring data and comprehensively examining the leakage, the following conclusion is obtained:

(1) From the overall varying pattern of leakage monitoring data, the trend of dam leakage is continuously deteriorating, which has imposed great threats to the safety of dam.

(2) From the numerical calculation, the seepage-proofing of face and anti-seepage curtain is significant, and the maintenance of face is quite critical to the safe operation of dam. The seepage flow velocity of downstream rockfill area is relatively great, and there is no high drainability requirement on it.

(3) For Baiyun hydropower dam, the river valley is narrow and both banks are high and precipitous, which poses higher requirement on the joint seal and panel structure around both banks. If they aren't properly handled, they would become the weak link of dam structure and seepage-proofing works, and this is also the reason of the serious leakage of similar project.

\section{References}

[1] Ministry of Water Resources Dam Safety Management Center. SL258-2000 Guidelines on Dam Safety Evaluation [S]. Beijing: China Water Conservancy and Hydropower Press, 2000.

[2] Gao Dashui, Cai Wei and Jiang Junjun, et. al. Examining Report of Leakage of Hydropower Dam of Baiyun, Bucheng County, Hunan Province [R]. Wuhan: Yangtze River Survey Planning and Design Research LLC., 2011.

[3] Hu Xiaolong, Yang Xieyun and Liu Yonghong et. al. Feasibility Study Report of Leakage Abatement Works of Hydropower Dam of Baiyun, Bucheng County, Hunan Province [R]. Wuhan: Yangtze River Survey Planning and Design Research LLC, 2014.

[4] Niu Xinqiang, Yang Qigui, Tan Jiexiong, et. al. Reservoir Dam Safety Evaluation [M]. Beijing: China Water Resources and Hydropower Press, 2007. 